\title{
Síndrome de Wolf-Hirschhorn: Microdeleción distal del brazo corto del cromosoma 4
}

\author{
JORGE A. AVIÑA F. ${ }^{1}$, DANIEL A. HERNÁNDEZ A. ${ }^{2}$ \\ 1. Dismorfología Pediátrica UMAE. Centro Médico Nacional de Occidente. Instituto Mexicano del Seguro Social IMSSS, \\ Guadalajara, México. \\ 2. Urgencias Médicoquirúrgicas, Cruz Verde. Servicios Médicos Municipales. Guadalajara, México.
}

\begin{abstract}
Wolf-Hirschhorn Syndrome: distal microdeletion of chromosome 4 short arm

Background: Wolf-Hirschhorn Syndrome is a genetic disease, in which the defect is a partial deletion involving the distal part of the short arm of chromosome 4. The clinical manifestations are craniofacial anomalies, delayed psychomotor development and neurological disorders. Objetive: Describe a clinical case of Wolf-Hirschhorn Syndrome, with specific description of craniofacial dysmorphological features. Case report: A female hypotonic infant with microcephaly and facial dysmorphism like "greek helmet": prominent glabela, ocular hypertelorism, epicanthal folds and marked broad-beaked nose, with pre and postnatal severe growth deficiency, mental retardation and seizures. Conclusions: The fluorescence in situ hybridization (FISH) karyotype revealed loss of genetic material at chromosome 4 short arm, with deletion in band $4 \mathrm{p} 15$ confirming the diagnosis. A case of probable de novo mutation with deletion of gene WHSC1 and other linked contiguous genes.

(Key words: Wolf-Hirschhorn Syndrome, chromosomal microdeletion).

Rev Chil Pediatr 2008; 79 (1): 50-53
\end{abstract}

\section{RESUMEN}

Introducción: El síndrome de Wolf-Hirschhorn es una rara enfermedad causada por una anormalidad cromosómica debida a una microdeleción distal del brazo corto del cromosoma 4; sus manifestaciones clínicas son: malformación craneofacial, retardo psicomotor severo, y alteraciones neurológicas diversas. Objetivo: Descripción de un caso clínico de Wolf-Hirschhorn, con énfasis en las alteraciones craneofaciales ilustrativas de la enfermedad. Cuadro clínico: Lactante hipotónico con microcefalia y rostro peculiar de "yelmo guerrero griego": frente amplia, glabela prominente, hipertelorismo, epicanto interno y nariz achatada; paciente con retardo en crecimiento pre y postnatal, retraso psicomotor y convulsiones. La confirmación del diagnóstico se logró con cariotipo de hibridación fluorescente in situ (FISH) mostrando microdeleción distal en brazo corto del cromosoma 4, banda p15. Conclusión: El caso puede corresponder a una mutación de novo con deleción del gen WHSC1 y otros vecinos pues es un síndrome de genes contiguos.

(Palabras clave: Síndrome de Wolf-Hirschhorn; microdeleción cromosómica).

Rev Chil Pediatr 2008; 79 (1): 50-53

Trabajo recibido el 06 de agosto de 2007, devuelto para corregir el 28 de agosto de 2007, segunda versión el 29 de septiembre de 2007, aceptado para publicación el 03 de noviembre de 2007.

Correspondencia a:

Jorge Arturo Aviña Fierro.

E-mail: avinafie@megared.net.mx 
El síndrome de Wolf-Hirshhorn es una rara enfermedad genética causada por la microdeleción distal del brazo corto del cromosoma 4. Las principales características son los rasgos faciales peculiares, crisis convulsivas y retardo psicomotor y del desarrollo. Su frecuencia de presentación es de 1 por cada 50000 nacidos vivos, presentando el doble de frecuencia en mujeres ${ }^{1}$. Fue descrito simultáneamente en 1965 en comunicaciones separadas por los doctores Ulrich Wolf ${ }^{2}$ y Kart Hirschhorn ${ }^{3}$, como un síndrome de microcefalia con cráneo peculiar en forma de yelmo guerrero griego; Zollino $\mathrm{M}$ et $\mathrm{al}^{4}$ en 2001, realizaron la delineación completa del síndrome: recién nacidos con bajo peso al nacimiento, hipotónicos, que presentan crisis convulsivas; el cuadro clínico está caracterizado por diversas malformaciones congénitas, microcefalia con asimetría craneal, hipertelorismo, fisuras palpebrales oblicuas, boca en forma de carpa, orejas displásicas de implantación baja y retrognatia; convulsiones de inicio temprano, cardiopatía congénita y retraso mental. La mitad de los pacientes tienen paladar hendido $\mathrm{y}$ los varones pueden presentar hipospadias y criptorquidia. Los criterios diagnósticos son: dismorfia facial característica en "yelmo griego", retardo del crecimiento y manifestaciones de déficit neurológico: retraso mental, hipotonía y convulsiones ${ }^{5}$.

\section{Caso Clínico}

Paciente de sexo femenino de 16 meses de edad, padres jóvenes sanos, no consanguíneos, madre 19 años, padre 24 años, toxicomanías negadas, no exposición a teratógenos. Producto de primera gestación a término, parto eutócico, peso al nacer $1800 \mathrm{~g}$, talla $45 \mathrm{~cm}$, Apgar 7, 8; desnutrida in útero y con hipotonía desde el nacimiento, hipoactiva con problemas de succión y deglución e importante reflujo gastroesofágico, requirió alimentación por sonda nasogástrica y gastroclisis. Cardiopatía congénita no cianótica compleja, con persistencia de conducto arterioso intervenida a la edad de 6 meses, posteriormente se detectó soplo holosistólico grado III de VI, comprobándose comunicación interauricular oval con dimensiones de 7,6 por
$5 \mathrm{~mm}$ con flujo de izquierda a derecha, requiriendo manejo médico continuo con digoxina, captopril y furosemida. $\mathrm{Al}$ año de edad presentó crisis convulsivas tónico-clónicas generalizadas frecuentes que se manejaron con fenobarbital y valproato sin lograrse control por lo que se añadió vigabatrina; un estudio de tomografía craneal mostró atrofia córtico-subcortical. El electroencefalograma mostró crisis intermitentes de alto voltaje $(2-3 \mathrm{~Hz})$ en ondas lentas intercaladas con espigas, detectadas principalmente en las zonas centroparietales y parietotemporales. El cariotipo del paciente con técnica de hibridación fluorescente in situ (FISH) reveló microdeleción distal del brazo corto en el cromosoma 4, banda p15. Los cariotipos de los padres fueron normales.

La exploración física mostró lactante microcéfalo con dismorfia craneofacial, frente amplia con glabela prominente, hipertelorismo ocular, fisuras palpebrales de inclinación oblicua descendente, ptosis palpebral, pliegues epicánticos internos, cejas escasas y ralas en forma de arco; boca de carpa con levantamiento del labio superior, arco de cupido marcado y comisuras labiales descendentes; filtrum corto y profundo (figura 1). Reborde orbital plano,

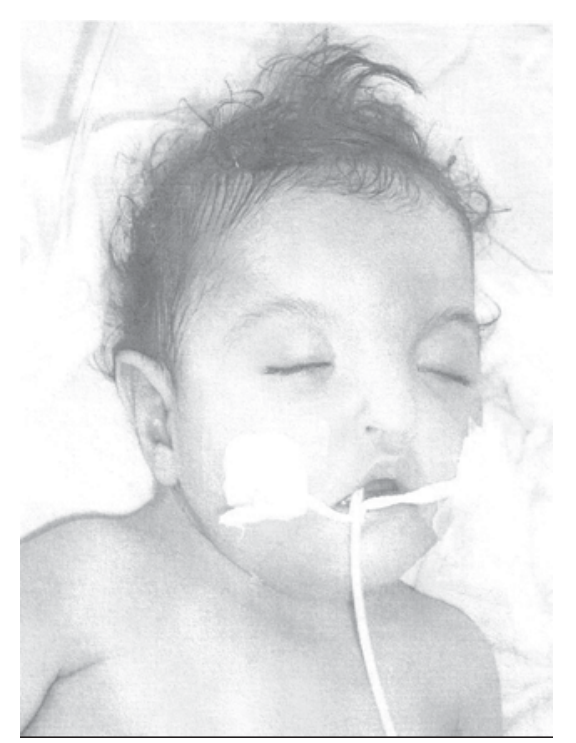

Figura 1. Lactante con microcefalia, hipertelorismo, fisuras palpebrales descendentes, epicanto interno, cejas en arco, boca de carpa. 


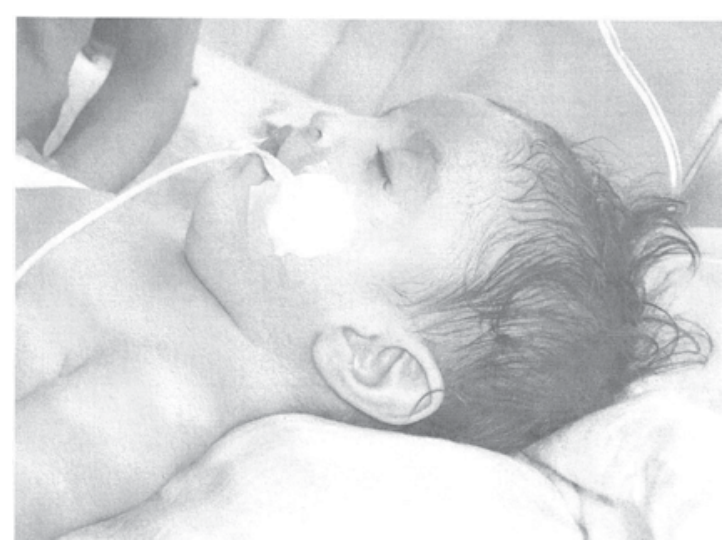

Figura 2. Reborde orbital plano, nariz pequeña, micrognatia, orejas de implantación baja y con rotación posterior.

puente nasal muy amplio, nariz pequeña con punta achatada y bulbosa, pabellones auriculares grandes en implantación baja y rotación posterior; hipoplasia maxilar, micrognatia y retrognatia, cuello corto y alado (figura 2). Cuerpo delgado y longilíneo, extremidades alargadas, manos con surco palmar transverso, dedos delgados y finos. Somatometría inferior al percentil 3 de su edad: talla $58 \mathrm{~cm}$ y peso de $4 \mathrm{~kg}$, perímetro cefálico muy reducido $(37 \mathrm{~cm})$, mano $7 \mathrm{~cm}$, pie $8 \mathrm{~cm}$, perímetro torácico $37 \mathrm{~cm}$, abdominal $36 \mathrm{~cm}$, segmento superior $34 \mathrm{~cm}$, e inferior $24 \mathrm{~cm}$. Todas estas medidas fuera de percentiles, correspondientes sólo a niños de 2 meses de edad.

\section{Discusión}

Se presenta un caso clínico representativo del síndrome de Wolf Hirschhorn, rara enfermedad causada por una deleción subtelomérica en el brazo corto del cromosoma 4, con alteración del gen WHSC1 ${ }^{6}$, que determina retardo del crecimiento pre y postnatal y las otras manifestaciones sindromáticas neurológicas y dismórficas ${ }^{7}$. El electroencefalograma mostró patrón eléctrico característico asociado a convulsiones mioclónicas y periodos de ausencias ${ }^{8}$. El cariotipo del paciente mostró microdeleción distal del brazo corto en cromosoma 4, banda p15; con padres sanos y sin antecedentes familiares de la enfermedad, la probabilidad es una mutación de novo ${ }^{9}$ causa más frecuente que com- prende el $75 \%$ de los casos (el 85\% de éstos son deleción proveniente del padre ${ }^{10}$; muy pocos casos (12\%) pueden tener alteraciones como un cromosoma 4 en anillo, mosaicismo, o traslocación esporádica no balanceada, pero nada de esto mostró el cariotipo; el resto (13\%) suelen ser casos con traslocación cromosómica parental $^{11}$ pero el cariotipo de los padres fue normal.

El diagnóstico diferencial se realizó con síndrome de Pitt-Rogers-Danks de herencia autosómica recesiva que cursa también con retraso pre y postnatal, microcefalia, facies característica, retardo del desarrollo, deficiencia mental grave y convulsiones ${ }^{12}$, y es una microdeleción del cromosoma 4, banda p16, considerado una variante de causa materna por segregación de translocación balanceada t $(4 ; 8)$. En Wolf Hirschhorn en cambio la causa suele corresponder a una impronta paterna con alteraciones en los loci D4S166 y D4S3327, y recientemente se ha reportado una nueva zona de afección D4S98-D4S16 denominada región WHSCR$2^{13,14}$. El pronóstico del paciente es desfavorable a corto plazo pues la supervivencia en la mayoría de los casos es aproximadamente de 2 años de vida, muriendo por complicaciones respiratorias y cardíacas; sólo un tercio sobrevive un tiempo mayor ${ }^{15}$. Este caso clínico es ilustrativo de las alteraciones morfológicas craneofaciales del síndrome de Wolf Hirschhorn y su difusión puede ayudar al diagnóstico de otros casos en Latinoamérica.

\section{Referencias}

1.- Moretti P, Ferrari M, Di Battista S, Di Battista C: The 4P-syndrome. Case description and literature review. Minerva Pediatr 2001; 53: 23-8.

2.- Wolf $U$, Reinwein $H$. Porsh R, Schroter R, Baitsch $H$ : Deficiency on the short arms of a chromosome No. 4. Humangenetik 1965; 1: 397-413.

3.- Hirschhorn K, Cooper H, Firschein IL: Deletion of short arms of chromosome 4-5 in a child with defects of midline fusion. Humangenetik 1965; 1: 479-82.

4.- Zollino M, Di Stefano C, Zampino G, Mastroiacovo P, Wright TJ et al: Genotype-phenotype correlations and clinical diagnostic criteria in Wolf-Hirschhorn syndrome. Am J Med Genet 2000; 94: 254-61.

5.- Battaglia A, Carey JC, Wright TJ: Wolf-Hirschhorn (4p-) syndrome. Adv Pediatr 2001; 48: 75-113.

6.- Bergemann $A D$, Cole F, Hirschhorn K: The etiology 
of Wolf-Hirschhorn syndrome. Trends Genet 2005; 21: 188-95.

7.- Iwanowski PS, Stengel-Rutkowski S, Anderlik L, Pilch J, Midro AT: Physical and developmental phenotype analyses in a boy with Wolf-Hirschhorn syndrome. Genet Couns 2005; 16: 31-40.

8.- Zankl A, Addor MC, Maeder-Ingvar MM, Schorderet DF: A characteristic EEG pattern in 4psyndrome: case report and review of the literature. Eur J Pediatr 2001; 160: 123-7.

9.- Lurie IW, Lazjuk GI, Ussova YI, Presman EB, Gurevich DB: The Wolf-Hirschhorn syndrome. I. Genetics. Clin Genet 1980; 17: 375-84.

10.- Tupler R, Bortotto L, Buhler E, Aikan M, Malik NJ, et al: Paternal origin of de novo deleted chromosome 4 in Wolf-Hirschhorn syndrome. J Med Genet 1992; 29: 53-5.

11.- Dallapiccola B, Mandich P, Bellone E, Selicorni A, Mokin $V$, et al: Parental origin of chromosome $4 \mathrm{p}$ deletion in Wolf-Hirschhhorn syndrome. Am J Med Genet 1993; 47: 921-4.

12.- Cabrera López JC, Marti Herrero M, Fernández Burriel M, Toledo L, de Andrés-Cofino R, Orera MA: Familial Pitt-Rogers-Danks: two new cases. Rev Neurol 2001; 33: 439-43.

13.- Zollino $M$, Lecce $R$, Fischetto $R$, Murdolo $M$, Faravelli $F$, et al. Mapping the Wolf-Hirschhorn syndrome phenotype outside the currently accepted WHS critical region and defining a new critical region, WHSCR-2. Am J Hum Genet 2003; 72: 590-7.

14.- Rodríguez L, Zollino M, Climent S, Mansilla E, LópezGrondona $F$, et al. The new Wolf-Hirschhorn syndrome critical region (WHSCR-2): a description of a second case. Am J Med Genet A 2005; 136: 175-8.

15.- Shannon NL, Maltby EL, Rigby AS, Quarrell OW: An epidemiological study of Wolf-Hirschhorn syndrome: life expectancy and cause of mortality. J Med Genet 2001; 38: 674-9. 